\title{
Hierarchical traffic control and management with intelligent vehicles*
}

\author{
L.D. Baskar, B. De Schutter, and H. Hellendoorn
}

If you want to cite this report, please use the following reference instead: L.D. Baskar, B. De Schutter, and H. Hellendoorn, "Hierarchical traffic control and management with intelligent vehicles," Proceedings of the 2007 IEEE Intelligent Vehicles Symposium (IV'07), Istanbul, Turkey, pp. 834-839, June 2007.

Delft Center for Systems and Control Delft University of Technology

Mekelweg 2, 2628 CD Delft

The Netherlands

phone: +31-15-278.24.73 (secretary)

URL: https: //www.dcsc.tudelft.nl

*This report can also be downloaded via https://pub.deschutter.info/abs/07_013.html 


\title{
Hierarchical Traffic Control and Management with Intelligent Vehicles
}

\author{
Lakshmi Dhevi Baskar, Bart De Schutter, and Hans Hellendoorn
}

\begin{abstract}
In this paper we first present a survey on traffic management and control frameworks that are based on intelligent vehicles (IV). This survey includes a short overview of IV-based traffic control measures such as cooperative adaptive cruise control, intelligent speed adaptation, and dynamic route planning. We also discuss various IV-based traffic management architectures such as PATH, Dolphin, Auto21 CDS, etc. Next, we propose a new integrated hierarchical IV-based traffic management control framework that combines the strong points of these architectures and extends them in various directions.
\end{abstract}

\section{INTRODUCTION}

In spite of well-planned road management schemes, sufficient infrastructures, and traffic rules for safe driving, modern societies still face the problem of traffic congestion due to the ever-increasing traffic demand, which in turn results in loss of time, fuel, and money. Building new roads could be a solution, but it is less feasible due to political and environmental concerns. An alternative would be to make efficient use of the existing infrastructure. In this context advanced technologies in the field of telecommunication and information systems offer an excellent opportunity to implement a next generation traffic control and management approach. This has led to the emergence of a new paradigm called "Intelligent Transportation Systems" (ITS) [1]. ITS incorporate intelligence in both roadways and vehicles, and aim at improving the traffic flow. Possible performance measures in this context are throughput, travel times, safety, fuel consumption, reliability of travel times, robustness, etc.

One way to improve traffic flow and safety of the current transportation systems is to apply automation and intelligent control methods to roadside infrastructure and vehicles. This gave rise to Automated Highway Systems (AHS), a component of advanced vehicle control systems that distributes the intelligence over the vehicles and the roadside infrastructure for better coordination of traffic network activities. So AHS shift the driver tasks from driver to the vehicle. These driver tasks include activities such as steering, changing gears, and making control decisions about speeds and safe headways that must be executed to reach the destination safely and comfortably. Autonomous control of driving tasks in ITS has the potential to substantially improve the traffic flow [2], [3]. By autonomous, we mean that the intelligent traffic management system takes partial or complete control of lateral and/or longitudinal movements of the vehicles.

L.D. Baskar, B. De Schutter, and H. Hellendoorn are with the Delft Center for Systems and Control, Delft University of Technology, Delft, The Netherlands. B. De Schutter is also with the Marine \& Transport Technology department of Delft University of Technology. $\{1 . \mathrm{d} . \mathrm{baskar}, \mathrm{j} . \mathrm{hellendoorn}\}$ @tudelft.nl, bedeschutter.info
The most interesting functionality that allows automated, hands-free operation is to arrange the vehicles in closely spaced groups called "platoons" [4]. Platooning subsumes smooth merging, lane changing, and splitting maneuvers. Within a platoon, vehicles can coordinate their activities using the communication technology. Spacing within a platoon (intra-platoon) is kept very small and the inter-platoon spacing is kept larger. Using the platooning concept, high speeds and short distances among vehicles can be maintained [2], [5]. This allows more cars to be accommodated on the network, resulting in an increased traffic flow. For driver comfort, the jerk produced by the vehicle acceleration or deceleration must not exceed a maximum value [6]. Furthermore, safety criteria should also be addressed. Although the automation concept for driving tasks promises to offer a better solution to traffic congestion problem, reliability of such systems must be proven and guaranteed in order to get public acceptance and adoption of this approach. Although the AHS program showed significant potential improvements in the traffic conditions, many consider AHS as a technology for the distant future. Hence, researchers started to concentrate on short-term programs aimed at enhancing intelligence in vehicles and driver assistance systems without relying on the infrastructure technology.

In this paper we first present a survey on intelligent vehicles (IV), and on IV-based control measures and IV-based traffic management frameworks. Next, based on the survey, we present a new IV-based traffic management framework that combines and extends existing frameworks. We combine the intelligence of roadside infrastructure and intelligent vehicle systems with automation. The approach we propose develops ITS-based control and management methods that integrate the additional control measures offered by in-vehicle telematics that support platooning with that of the roadside infrastructure to substantially improve traffic performance in terms of safety, throughput, and environment.

\section{INTELLIGENT VEHICLES}

Intelligent Vehicles (IV) enable a next generation approach for obtaining a more efficient driver-vehicle operation [7]. An IV system senses the environment around the vehicle and strives to achieve more efficient vehicle operation by assisting the driver (advisory/warning) or by taking complete control of the vehicle (automation). IV systems can be applied in all types of vehicles such as cars, buses, and trucks. We can divide IV application areas into three categories depending on the level of support provided to the driver:

- Advisory systems use optic or acoustic systems to provide an advisory/warning to the drivers. 
- Semi-autonomous systems use haptic (meaning "based on the sense of touch") measures to assist/take partial control of the vehicle. An example of haptic control is an "active accelerator" for intelligent speed adaptation (ISA): When the speed limit is exceeded, the driver gets a counter-force feedback on the accelerator. So if he wants to exceed the speed limit, he has to exert more pressure on the pedal to override this counter force.

- Fully autonomous systems take complete control of vehicle operation.

IV-based control measures offer lateral, longitudinal, or integrated control systems for improving safety, operational efficiency, and driving comfort [7]. These measures, when combined with autonomous control, could help to reduce the reaction time of the driver and vehicle, and allow to achieve a decreased minimum safe distance between vehicles which in turn leads to an improved traffic throughput.

\section{A. IV control measures and traffic control}

If the vehicle parameters and state variables (such as speed, acceleration, desired destination, etc.) are allowed to be exchanged effectively among the vehicles in a platoon using communication technologies, then the vehicle distance and speed can be controlled more closely. We will now discuss in more detail the following IV technologies:

- Cooperative Adaptive Cruise Control (ACC),

- Intelligent Speed Adaptation (ISA),

- dynamic route planning.

1) Cooperative ACC: Controlling longitudinal motion and maintaining a minimum time gap between two vehicles has been considered as one of the fundamental tasks of driving. Many vehicles have already been deployed with partial automation of this task by the use of the standard conventional cruise control [8].

An ACC system is a radar-based system that is able to sense the immediate vehicle in front on the lane, and automatically adjust the speed of the equipped vehicle to match the speed of the preceding vehicle to maintain a safe inter-vehicle distance for avoiding collisions [9], [10]. By adjusting, we mean that the equipped vehicle slows down when the vehicle in front is traveling slowly by reducing gas or by active braking, and afterwards maintains a specific time gap that is chosen by the driver in advance. If there is no vehicle in front, then ACC controller retains the preset speed that was selected by driver.

Cooperative ACC is a further enhancement of ACC systems that utilizes existing communication technologies (e.g., wireless technologies) to obtain real-time information about the speed, acceleration, etc. of the preceding vehicle to maintain a safe and smooth driving. Possible parameters and variables that can be exchanged are current speed, position, abrupt braking maneuvers, and braking capability and acceleration. Cooperative ACC equipped vehicles can exchange the information much quicker and allow to set the safe minimum headway as small as $0.5 \mathrm{~s}$. Hence, with reduced headways between vehicles, traffic flow can be improved.
2) ISA: A standard speed limiter is a system that restricts the speed of the vehicle when the driver tries to exceed the maximum allowed driving speed, and is independent of a speed limit specific to a road environment. When the speed limiter is enhanced with the intelligence to consider the speed limit restrictions, and when it is able to adjust the maximum driving speed to the speed limit specified by the roadside infrastructure, and to provide feedback to the driver when that speed limit is exceeded, then we get the technology called Intelligent Speed Adaptation (ISA) [11], [12].

ISA systems can be categorized along several dimensions. The characterization could be based on the extent to which the driver is going to follow the feedback of the system when the speed limit is exceeded. This results in advisory systems, voluntary systems, and mandatory systems.

Another characterization for ISA systems can be made based on the speed limit itself and they are classified as fixed or dynamic speed limits. In the fixed case, the driver is informed about the speed limit, which could be obtained from a static database. The dynamic speed limit takes account of current road conditions such as bad weather, slippery roads, or major incidents before prescribing the speed limit.

3) Dynamic route planning: Nowadays, many individual vehicles are equipped with a route guidance or navigation system. Generally, a route guidance system advises a driver about the "best" route he can take to reach his requested destination [13]-[15]. This mainly depends on the vehicle's current location. Using GPS, we can precisely determine the vehicle's position. This information can be looked up a digital road map. The digital map can then be used to determine the possible routes to reach the destination. Routes recommendations may be calculated within the equipped vehicle or communicated to the vehicle from the local traffic center. When the possible routes are computed based on the average conditions such as geographical locations and road maps, then this scheme is referred to as static route guidance system. If the existing traffic conditions such as traffic jams, dynamic speed limits, and frequent updates of travel times based on real-time traffic are taken into account while computing the route recommendations, then this is called dynamic route planning system.

\section{CONTROL Frameworks AND ARCHItectures}

We now focus on control architectures that have been developed for linking the roadside infrastructure and automated vehicles. A well-known control framework, called PATH was proposed by the University of Berkeley. This framework served as a guideline for a several other ITS few architectures, which are also discussed in this section.

\section{A. PATH framework}

The PATH architecture mainly focuses on automatic vehicle control and coordinates both roadside/vehicle and intervehicle activities. This framework assumes that the traffic is organized in platoons in order to improve traffic flow and to increase safety [2], [4], [16]. The PATH framework assumes 


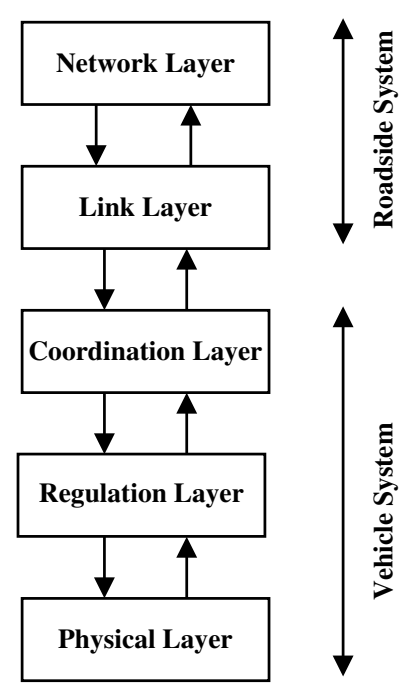

Fig. 1. PATH architecture

that a traffic network consists of many interconnected highways. Highways can be again divided into links (about $5 \mathrm{~km}$ long). A link is subdivided into sections (about $1 \mathrm{~km}$ long) and these sections consist of lanes. The designed framework is a hierarchical structure with the control of automated highway system distributed into five self-organized functional layers as shown in Figure 1:

1) Network layer: The network layer is the top layer in the hierarchy. At this layer, the controller computes control actions that optimize the entire network. Its task is to assign a route for each vehicle that enters the highway ensuring that the capacity of each potential route is utilized properly. There is one network layer for each highway network.

2) Link layer: The second layer in the control hierarchy is the link layer. This layer operates at the roadside and has a controller located per each link. Each link controller receives commands from the network layer and based on these commands, the link controller calculates the maximum platoon size, and the optimum platoon velocity for each section, and it also sets the path (which lane to follow) for each vehicle to reach its destination as quickly as possible.

3) Coordination layer: From the coordination layer onwards, the control actions are concerned with individual vehicles rather than roadside infrastructure. A coordination controller resides within each vehicle, receives the commands from the link layer, coordinates with other neighboring platoons using protocols, and checks which maneuvers (like lane change, split, or merge) have to be performed on a platoon to assist a vehicle in a platoon to achieve the path trajectory in such a way that the target platoon size and speed specified by the link layer are maintained. Only the leader of the platoon or a free agent (i.e., a vehicle that does not (yet) belong to a platoon) can initiate the maneuvers with other platoons. The follower vehicles can only make requests to the leader to accomplish their path/goal.

4) Regulation layer: The regulation layer within each vehicle executes the tasks specified by the coordination layer by converting them into throttle, steering, and braking inputs for the actuators of the vehicle. This controller uses feedback control laws to execute the lateral and longitudinal maneuvers and also notifies the coordination layer in case of any failures or unsafe outcomes of the maneuver.

5) Physical layer: The physical layer of each vehicle is the bottom layer of the hierarchy and involves the actual dynamics of the vehicle. This layer receives information from the regulation layer and also sends information about the speed, acceleration, and engine state of the vehicle to the regulation layer.

\section{B. Dolphin framework}

The Japanese Dolphin framework developed in [17] is similar to the PATH architecture. This framework considers platoon-based/cooperative driving for automated vehicles using sensor systems, and inter-vehicle communication technologies. This framework is mainly intended to study inter-vehicle communication technologies and to control the platoon maneuvers without incorporating intelligence in the roadside infrastructure. So this architecture assumes that all vehicles can drive autonomously without expecting intelligence from the roadside infrastructure. It is composed of three layers as shown in Figure 2:

1) Traffic control layer: The traffic control layer is the top layer and it is divided internally into two parts namely, logical and physical. The physical part includes the roadside ITS equipment like sign boards, road-vehicle communications, ramp metering, and traffic signals. The logical part consists of laws, rules, and common sense.

There is only one traffic control layer common to all the vehicles and is related to the roadside infrastructure. The traffic control layer determines criteria that will be common to all the neighboring vehicles and sends them to the next layer. E.g., in an automated driving, the criteria could be to maintain a small inter-vehicle distance between the followers in a platoon, and to provide an autonomous driving capability for the leaders in a platoon.

2) Vehicle management layer: The vehicle management layer, which resides in each vehicle, receives its movement criteria from the traffic control layer via road-vehicle communication and also considers the messages from the neighboring vehicles via inter-vehicle communication and the data received from the basic vehicle control layer. This layer determines the movements of the individual vehicle under platoon-based driving in such a way that these commands are satisfied. Lateral and longitudinal control for the platoon leader or free agent are determined by this layer using, e.g., GPS and a digital map. The vehicle management layer controllers in the follower vehicles determine both the lateral and longitudinal control commands.

3) Vehicle control layer: The vehicle control layer within each vehicle is the lower layer. The basic function of this layer is to sense the states and the conditions ahead of the vehicle such as vehicle speed and acceleration, to send this information to the vehicle management layer, and to receive commands from the above layer for its steering actions. 


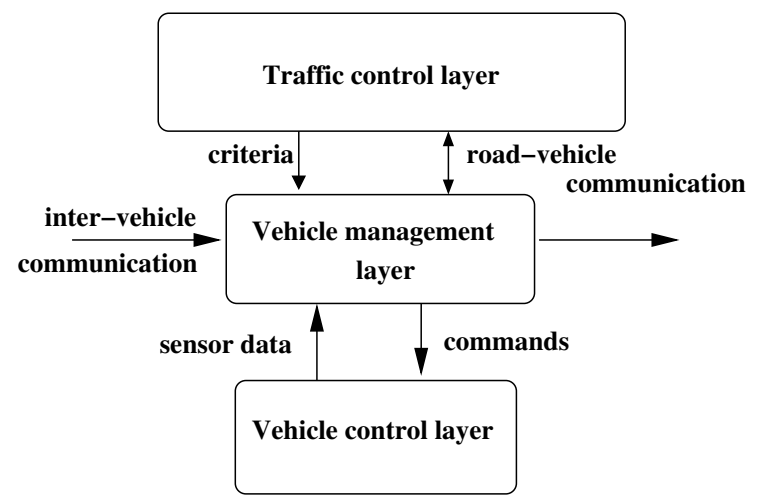

Fig. 2. Dolphin architecture for cooperative driving

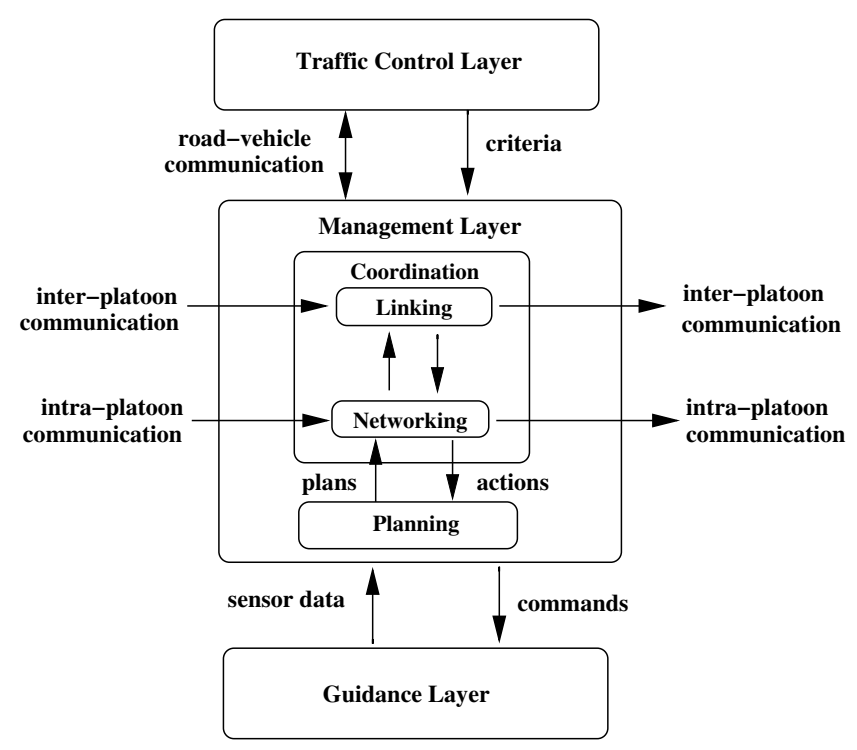

Fig. 3. CDS architecture for cooperative driving

\section{Auto21 CDS framework}

The Collaborative Driving System (CDS) framework [18], [19] is mainly inspired by the PATH and Dolphin architectures. The CDS architecture uses cooperative ACC technologies to support platoon-based driving. The CDS framework aims to use an inter-vehicle coordination system that can ensure stability among the vehicles in a platoon. As shown in Figure 3, this framework has three layers:

1) Traffic control layer: The traffic control layer is on the roadside infrastructure and provides suggestions (not commands) to the lower layer such as speed, platoon sizes, lane changes, etc. To transfer this information, the traffic control layer interfaces with the linking module (located in management layer) which resides inside each vehicle. The linking module is also used by the traffic control layer to obtain observations on the traffic situation [20].

2) Management layer: The management layer resides within each vehicle and is responsible for determining the movements of each vehicle using the information received from the guidance layer, the traffic control layer, and vehicle coordination constraints through inter-vehicle communica- tion. This layer is subdivided into coordination and planning sublayers, which work cooperatively:

- The coordination sublayer has a linking module that communicates with the traffic control layer to receive suggestions on the lane change actions and that maintains the inter-platoon activities. Once the linking module has made a choice about the action to perform, the maneuvers are executed (merge or split from a platoon) by the networking module which handles the intraplatoon coordination. The networking module along with the planning sublayer makes cooperative plans to execute the intra-platoon maneuvers.

- The planning sublayer is responsible for making the plans to execute the maneuvers inside the platoons. In order to do this planning, this sublayer communicates with other vehicles in the platoon using the networking module and also receives data from the guidance layer.

3) Guidance layer: The guidance layer senses the states of the vehicle and sends the vehicle parameters to the management layer and also receives commands from the layer above for throttle, braking and steering actions.

\section{RELATED INITIATIVES AND PROJECTS}

\section{A. CarTALK2000}

CarTALK is a European project that focuses on driver assistance systems based on inter-vehicle communication [21]. Its main objective is to develop a cooperative driver assistance system with self-organizing ad-hoc radio network as communication basis. To achieve a suitable communication system, algorithms for flexible and ad-hoc radio networks with highly dynamic topologies are developed in this project.

\section{B. Safespot}

The objective of the European research project SAFESPOT [22] is to improve road safety using intelligent vehicles and intelligent roads. Th SAFESPOT safety margin assistant can detect dangerous situations in advance and can provide awareness of the surrounding environment to the driver. The safety margin assistant is based on vehicle-tovehicle and vehicle-to-infrastructure communication.

\section{PreVent}

PReVENT [23] is a European automotive industry activity. Its main focus to develop preventive applications that can improve the road safety. These safety applications use invehicle systems to anticipate the nature and significance of the danger and to help the drivers to avoid accidents.

\section{CVIS}

CVIS (Cooperative Vehicle-Infrastructure Systems) [24] is a European research project that aims to design, develop, and test technologies that allow communication between the cars and with the roadside infrastructure, which improves road safety and efficiency, and reduces environmental impact. This project allows drivers to influence the traffic control system directly and also to get information about the quickest route to their destination, speed limits on the road, as well as warning messages via wireless technologies. 


\section{A NEW FRAMEWORK FOR COOPERATIVE IV-BASED TRAFFIC MANAGEMENT}

Based on an extensive survey of IV-based traffic management framework the main components and results of which are reported above, we now propose a new IV-based framework that combines several of the strong points of existing architectures and that further enhances and extends them in several directions. The objective of the framework is to integrate the intelligence of roadside infrastructure and IV systems with automation. We propose a framework that distributes the intelligence between roadside infrastructure and vehicles, that assigns the traffic control actions based on platoons rather than on segment concept, and that uses the IV-based control measures to improve the traffic flow. We also incorporate the advantages of other frameworks such as CarTalk, SafeSpot, Prevent, CVIS, etc. The main improvements and extensions of the new framework are:

- We allow vehicles to communicate with each other and with the roadside infrastructure in an integrated way.

- We consider both in-vehicle IV-based traffic control measures (e.g., ISA, ACC) and roadside traffic control measures (e.g., ramp metering, variable speed limits, dynamic route guidance, shoulder lanes openings) and apply them in an integrated way.

- The platoon size is one of the variables that are optimized and it can range from 1 to a very large number, depending on the current traffic conditions, the traffic network, and the given traffic performance criterion.

- The framework is integrated with a model-based predictive control strategy that determines optimal traffic control measures in a receding horizon approach.

- The framework is also suited for inter-urban and even intra-urban traffic networks.

The new framework is now presented in more detail.

\section{A. IV-based control framework}

The goal of the proposed framework is to use the additional measures and control handles offered by IVs and to implement them in a hierarchical roadside/vehicle traffic management structure to substantially improve traffic performance in terms of safety, throughput, reliability, environment, and robustness. The framework mainly aims at a multi-level control structure with local controllers at the lowest level and one or more higher supervisory control levels and uses a combination and integration of techniques from computer science and control engineering in order to obtain coordination at and across all control levels.

Our framework is inspired by the AHS platoon concept and takes the opportunity of IV-based control measures to implement a next-level of traffic control and management, which shifts away from the rather global road-side traffic management to a more vehicle-oriented traffic management. We will consider both roadside-vehicle traffic management and interaction, and inter-vehicle traffic management and coordination. Although the PATH framework includes both roadside infrastructure and vehicles, much of the research

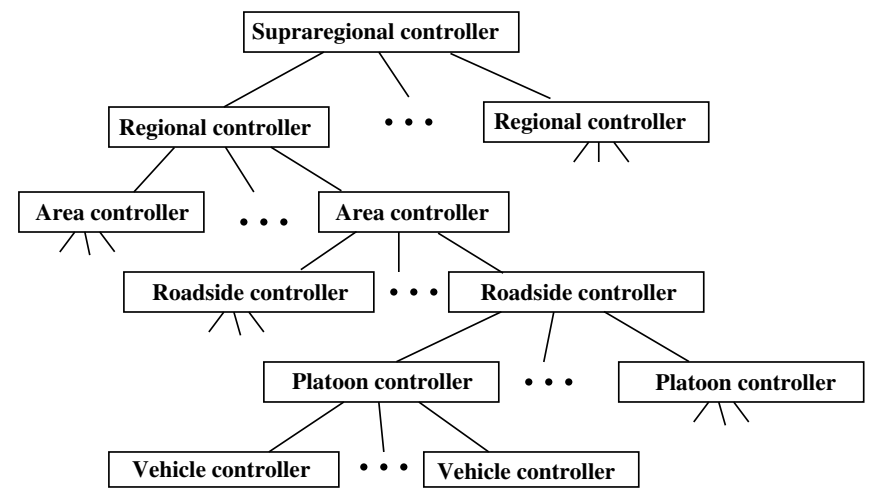

Fig. 4. New IV-based framework

work was carried on the vehicle control side. The roadside controllers determine the activities that need to be carried out in different sections. However, when the platoon size is allowed to be long enough, then it might be difficult for the roadside controller to assign the activities as the platoon resides in between two sections, and also for the vehicle controller to complete the activity within the specified space. It might also apply for a platoon of size one, where the vehicle has to adapt to the speed change in each section irrespective of its activity completion. For this reason, we use platoon-based roadside controllers (so without sections).

In order to be able to deal with large-scale traffic networks and also to increase flexibility and robustness, we consider a hierarchical control structure with several layers each of which consist of several controllers (see Figure 4):

1) Higher level controllers: Higher level controllers (area, regional, and supraregional) provide network-wide coordination of the lower-level and middle-level controllers. E.g., there could be an area controller to control/supervise the activities of a collection of roadside controllers. In turn, a group of area controllers could be supervised by regional controllers and this hierarchy grows as shown in Figure 4.

2) Roadside controller: The roadside controller in the hierarchy uses IV-based control measures to improve the traffic flow. The controller assigns desired speeds for each platoon (ISA), safe distances between platoons (Cooperative ACC), metering values on the on-ramps and off-ramps (ramp metering), desired platoon sizes, provides dynamic route guidance for the platoons and also instructs for merges, splits, and lane changes of platoons. This layer may control a part of a highway, an entire highway, or a collection of highways.

3) Platoon controller: The platoon controller is responsible for control and coordination of each vehicle in the platoon. The platoon controller receives commands from the roadside controller and is mainly concerned with actually executing the inter-platoon maneuvers such as merges, splits, and lane changes and intra-platoon activities such as maintaining safe inter-vehicle distances and acceleration for accomplishing the tasks assigned by the roadside controller.

4) Vehicle controller: The vehicle controllers present in each vehicle are the low-level controllers of the architecture. They receive commands from the platoon controllers (e.g., 
set-points or reference trajectories for speeds, paths, headways) and translate these commands into control signals for the vehicle actuators (throttle, braking, and steering).

\section{B. Integrated in-vehicle and roadside control measures}

In the new framework we integrate individual IV-based traffic control measures such as cooperative ACC, ISA, dynamic route guidance, with roadside traffic control measures such as ramp metering, traffic signals, lanes closures, shoulder lane openings, etc. The actual control strategy could then make use of a model-based predictive control approach such as model predictive control (MPC) [25]. MPC has originated in the process industry and has also been extended to conventional road-side based non-IV traffic management [26], [27]. This approach can also be extended to the proposed new IV-based traffic management framework.

Note that the proposed framework is not limited to freeway networks only, but also includes inter-urban and even intraurban traffic networks. Of course, due to more fragmented nature of the intra-urban and inter-urban networks, optimal platoon sizes in these latter environments will be much smaller. As the platoon size is also one of the control parameters in our framework this can be taken into account.

\section{Contributions of our approach}

The main contributions of the proposed framework are:

- Our approach integrates the roadside infrastructure and the platoon of vehicles as done in the AHS framework, but the roadside controller calculates optimal control actions for each platoon rather than to each vehicle in a section using both IV-based and roadside measures.

- Our framework can determine and assign optimal platoon sizes going from 1 to a very large number.

- The hierarchical structure with one or more supervisors added to the existing AHS architecture could prove to be an advantage of our framework. Large-scale traffic networks can be considered and also coordination of the mid-level and low-level controllers for such a wide network can be obtained by our framework.

- Most AHS architectures focus their implementation exclusively on highways. The framework we propose can be implemented on both highways and urban roads.

\section{CONCLUSIONS AND FUTURE WORK}

We have proposed a framework that combines IV-based measures and roadside infrastructure for implementing nextlevel traffic management. Topics for future work include: determining appropriate traffic models for this IV-based framework, defining the benchmarks, implementing this framework on a small-scale setup, developing software tools, and analyzing the trade-offs between computational complexity and efficiency for this framework.

\section{ACKNOWLEDGMENTS}

Research supported by the STW VIDI project "MultiAgent Control of Large-Scale Hybrid Systems" (DWV.6188), the BSIK projects "Transition Sustainable Mobility (TRANSUMO)" and "Next Generation Infrastructures (NGI)", and the Transport Research Centre Delft.

\section{REFERENCES}

[1] J. M. Sussman, "Intelligent vehicle highway systems: Challenge for the future," IEEE Micro, vol. 1, no. 14-18, pp. 101-104, June 1993.

[2] P. Varaiya, "Smart cars on smart roads," IEEE Trans. on Automatic Control, vol. 38, no. 2, pp. 195-207, Feb. 1993.

[3] S. Shladover, C. A. Desoer, J. K. Hedrick, M. Tomizuka, J. Walrand, W. B. Zhang, D. H. McMahon, H. Peng, S. Sheikholeslam, and N. McKeown, "Automatic vehicle control developments in the PATH program," IEEE Trans. on Vehicular Technology, vol. 40, no. 1, pp. 114-130, 1991.

[4] M. Broucke and P. Varaiya, "The automated highway system: A transportation technology for the 21st century," Control Engineering Practice, vol. 5, no. 11, pp. 1583-1590, Nov. 1997.

[5] K. Li and P. Ioannou, "Modeling of traffic flow of automated vehicles," IEEE Trans. on Intelligent Transp. Systems, vol. 5, no. 2, pp. 99-113, June 2004.

[6] A. Kanaris, P. Ioannou, and H. Fu-Sheng, "Spacing and capacity evaluations for different AHS concepts," in Proc. 1997 American Control Conf., Albuquerque, 1997, pp. 2036-2040.

[7] R. Bishop, Intelligent Vehicles Technology and Trends, Artech House, 2005.

[8] M. J. Richardson and D. Smith, "Design of the driver interface for autonomous intelligent cruise control," in IEE Colloquium on Design of the Driver Interface, London, 1995, pp. 7/1-7/4.

[9] L. C. Davis, "Effect of adaptive cruise control systems on traffic flow," Physical Review E, vol. 69, pp. 1-8, 2004.

[10] S. Darbha and K. R. Rajagopal, "Intelligent cruise control systems and traffic flow stability," Transp. Research Part C, vol. 7, no. 6, pp. 329-352, Dec. 1999.

[11] J. Blum and A. Eskandarian, "Managing effectiveness and acceptability in intelligent speed adaptation systems," in Int. Conf. on Intelligent Transp. Systems, Toronto, Sept. 2006, pp. 319-324.

[12] L. S. Comte, "New systems: new behaviour?" Transp. Research Part $F$, vol. 3, no. 2, pp. 95-111, May 2000.

[13] D. Watling and T. van Vuren, "The modelling of dynamic route guidance systems," Transp. Research Part C, vol. 1, no. 2, pp. 159182, June 1993.

[14] M. McDonald, N. B. Hounsell, and S. R. Njoze, "Strategies for route guidance systems taking account of driver response," in Proc. 6th Vehicle Navigation and Information Systems Conf., Seattle, July 1995, pp. $328-333$.

[15] H. Shimizu, M. Kobayashi, and Y. Yonezawa, "A route guidance system of a traffic network," in Proc. 34th SICE Annual Conf., Hokkaido, July 1995, pp. 1549-1552.

[16] R. Horowitz and P. Varaiya, "Control design of an automated highway system," Proceedings of the IEEE: Special Issue on Hybrid Systems, vol. 88, no. 7, pp. 913-925, July 2000.

[17] S. Tsugawa, S. Kato, K. Tokuda, T. Matsui, and H. Fujii, "An architecture for cooperative driving of automated vehicles," in Proc. IEEE Intelligent Transp. Symposium, Dearborn, 2000, pp. 422-427.

[18] "http://www.damas.ift.ulaval.ca/projets/auto21/en/index.html."

[19] "http://www.auto21.ca/home_e.html."

[20] S. Hallé, B. Chaib-draa, and J. Laumonier, "Car platoons simulated as a multiagent system," in Proc. 4th Workshop on Agent-Based Simulation, Montpellier, 2003, pp. 57-63.

[21] D. Reichard, M. Miglietta, L. Moretti, P. Morsink, and W. Schulz, "Cartalk2000 - safe and comfortable driving based upon inter-vehicle communication," in Proc. IEEE Intelligent Vehicle Symp., Karlsruhe, 2002, pp. 545-550.

[22] "http://www.safespot-eu.org/.",

[23] "http://www.prevent-ip.org/."

[24] "http://www.cvisproject.org/."

[25] E. Camacho and C. Bordons, Model Predictive Control in the Process Industry, Springer, 1995.

[26] A. Hegyi, B. De Schutter, and H. Hellendoorn, "Model predictive control for optimal coordination of ramp metering and variable speed limits," Transp. Research C, vol. 13, no. 3, pp. 185-209, June 2005.

[27] T. Bellemans, B. De Schutter, and B. De Moor, "Model predictive control for ramp metering of motorway traffic: A case study," Control Engineering Practice, vol. 14, no. 7, pp. 757-767, July 2006. 